\title{
Transient Aspects of MRP Theory - Abridged
}

\author{
Robert W. Grubbström
}

\begin{abstract}
MRP theory concerns production-inventory systems, in which produced items are made up of sets of sub-items, either produced or purchased (imported into the system) and required to be available a lead-time before the product is completed. The hierarchical dependence of relations between items is captured by the use of input matrices from Input-Output Analysis, the necessary advanced timing by applying the methodology of Laplace transforms, and the production-economic consequences by the net present value (NPV). This theory has been developed for about 40 years, but little attention has been given to transient aspects, i.e. when there happens to be an initial available inventory of items usable in future production. In this paper, we attempt to highlight the theoretical consequences from having a positive initial available inventory.
\end{abstract}

Keywords: Laplace transform methodology; Input-Output Analysis; NPV; Production Economics; MRP Theory

\section{INTRODUCTION}

Material Requirements Planning (MRP) is a management information system providing a base for production decisions, when what is manufactured has a complex structure and when lead times are important features. MRP is a control system (mainly) dealing with the production of assembled products, made up of items, such as subassemblies, components, raw materials. Taking processing times into account, required raw materials and sub-components will be needed ahead of times at which the production of a particular item is completed. The MRP system calculates the amounts of produced and purchased items needed at different times and proposes a plan for when this production is to be carried out, taking these lead times into account.

MRP Theory is a theoretical body combing MRP and Input-Output Analysis with Laplace Transform methodology aiming at determining an optimal production schedule for a production-inventory system. In this paper we shall confine ourselves to assembly systems (convergent systems), in which end items (to be sold or delivered from the system), but MRP Theory has been developed and extended to other types of production such as extractive processes and transportation/distribution systems (divergent production systems), and also to recycling (reverse logistics) systems, including for instance disassembly processes. For overviews, cf. [1], [2] and for applications, e.g. [3], [4].

The first hard-cover book on Material Requirements Planning [5] was authored in 1975 by Joseph A. Orlycky, 1922-1986. In his foreword (p. ix) was stated that "subject of material requirements planning has been neglected in hardcover literature and academic curricula ....one of the reasons for this situation is the subject's position outside the scope of quantitative analysis, and the view of it as being 'vocational' rather than 'scientific'." This rather gloomy comment was a challenge for many, and this current paper is yet another voice in this choir.

Two decades before that in the first issue of Management Science, Andrew Vazsonyi [6] had presented his result that the Bill-of-Materials (BOM) for an assembled product could well be written in mathematical form as a matrix the "Next Assembly Quantity Matrix", here denoted $\boldsymbol{H}$. When taking into consideration total requirements of all subassemblies, components, and materials, so on, that are needed for dispatching a batch of products (to be exported from the system), this was derived as the corresponding matrix $(\boldsymbol{I}-\boldsymbol{H})^{-1}$, describing the number of items needed for exporting one unit of an end product and coined by Vazsonyi as the "Total Factor Requirement" matrix.

These two matrices were also included in his book "Scientific Programming in Business and Industry" published three years later [7]. However, lead-times could not yet be captured in his treatment. And Nobel Laureate Tjalling C. Koopmans [8] (Koopmans 1951) and others before him recognised the commonality between Nobel Laureate Wassily W. Leontef's Input-Output Analysis [9], [10] with a macroeconomic and empirical touch, and the base for Vazonyi's s treatment, then for a time, named Activity Analysis. But with Input-Output Analysis, it became clear that $\boldsymbol{H}$ was the input matrix, and $(\boldsymbol{I}-\boldsymbol{H})^{-1}$ was the Leontief inverse of Input-Output Analysis.

In 1992 Grubbström and Ovrin published an article [11] using the z-transform (the discrete-time transform corresponding to the Laplace transform) to assembly processes. Since then, there have been many developments in what we now call "MRP Theory". By the help of, in particular, Professors Ludvik Bogataj and Marija Bogataj, this theory has been extended to transportation and recycling systems, having a quite different structure compared to assembly systems, cf. [12-13]. The optimal production plan $\boldsymbol{P}^{*}$ maximising NPV for any assembly system was stated and a solution method presented in [14].

We have organised the material in this paper as follows. The next section is devoted to the main parts of the Laplace transform method that are applied in MRP Theory. Section 3 develops our main results, which is followed by a section dealing with the economic value of having initial stocks. A numerical example follows in Section 5. This paper, as its title suggests, is an abridged version of this research. One important set of findings omitted below concerns initial backlogs, which comprise a parallel set of initial state 
variables compared to initial stocks, and which have not found space to be dwelled upon in this paper. Neither do we here allow for the possibility of initially outstanding orders, later to be fed into available inventory.

\section{DIGRESSION ON THE LAPLACE TRANSFORM}

The Laplace transform is named after the French mathematician, astronomer and physicist Pierre-Simon Laplace, 1749-1827. A forerunner in this methodology was the Swiss mathematician Leonard Euler, 1707-1783. The Laplace transform was originally used for solving differential equations and investigating stability properties of dynamic systems in electrical and mechanical engineering and astronomy, and in probability theory as a moment-generating function. More recent fields of application have been finance, production economics and risk preference theory, see [1622].

Deakin [23-25] offers an overview of the historical development of its use. For its general theory and method, see e.g, [26-29]. One of the first applications to productioneconomic problems was made by Nobel Laureate Herbert A. Simon [30]. where he applied the transform method to controlling a simple production-inventory system with a time lag in production.

The Laplace transform translates a function of time $x(t)$ into its transform, being a different function $\tilde{x}(s)$ of a frequency $s$. In all standard applications, in which certain regularity conditions are valid for $x(t)$, there is a one-to-one relationship between these two functions, so given the time function, all of its properties are captured in its transform, and vice versa.

The (unilateral) transform considers functions only existing for non-negative values of $t$, and is defined by

$£\{x(t)\}=\tilde{x}(s)=\int_{t=0}^{\infty} x(t) \mathrm{e}^{-s t} \mathrm{~d} t$

showing two alternatives for the notation of the transform. For the bilateral transform, applied e.g. in probability theory, the integration covers the entire time axis $\int_{t=-\infty}^{\infty} x(t) \mathrm{e}^{-s t} \mathrm{~d} t$, and $t$ is often interpreted with a different dimension than time, but in our applications in MRP Theory, only the unilateral transform is used. In general, the frequency $s$ is a complex variable $s=\sigma+i \omega$, with $\sigma$ being the real part of $s$, and $i \omega$ the imaginary part, where $i$ is the imaginary unit $i=\sqrt{-1}$. Translating the transform from the frequency domain back into its time function may be written $x(t)=£^{-1}\{\tilde{x}(s)\}$, where the operation $£^{-1}\{\cdot\}$ defines the inverse transform. A method for finding $x(t)$ from a given $\tilde{x}(s)$, is given by the integral

$x(t)=\mathfrak{f}^{-1}\{\tilde{x}(s)\}=\frac{1}{2 \pi i} \int_{s=\beta-i \infty}^{\beta+i \infty} \mathrm{e}^{s t} \tilde{x}(s) \mathrm{d} s$,

where the integration takes place in the complex $(\sigma, \omega)-$ plane along a vertical line $\sigma=\beta$, where $\beta$ is chosen such that the integral converges. To evaluate this integral, Cauchy's Residue Theorem may be used, Augustin Louis Cauchy, 1789-1857, being a disciple of Laplace.

In this article, in particular, we will make use of the following theorems of the Laplace transform.

Time differentiation

$£\left\{\frac{\mathrm{d} x(t)}{\mathrm{d} t}\right\}=s \tilde{x}(s)$.

There are alternative conventions concerning this notation, depending on whether or not the possible step at $t=$ 0 is included, but we prefer the convention in (3), which includes this step in the derivative.

Time integration

$£\left\{\int_{0}^{t} x(\tau) \mathrm{d} \tau\right\}=£\{\bar{x}(t)\}=\frac{\tilde{x}(s)}{s}$.

The bar notation (middle member of (4)) for cumulate flows will be used throughout this paper.

Final value (assuming this limit exists)

$\lim _{t \rightarrow \infty} x(t)=\lim _{s \rightarrow 0} s \tilde{x}(s)$.

Time translation (moving the time function uniformly along the time axis)

$£\{x(t-T)\}=\mathrm{e}^{-s T} \tilde{x}(s)$,

which holds as long as $t \geq T$, so $x(t)$ must be zero for $t<T$. When a function $x(t)$ is moved forwards in time, we have $T>0$, and this formula holds defining $x(t-T)$ to be zero for $t<T$. However, when we move backwards in time and $T<0$, then the function might cross $t=0$ and it then would become truncated. So the formula assumes always that $x(t-T)=0$ for $t<T$.

Important in MRP Theory is the Dirac impulse (the impulse function) written $\delta(t-T)$. It is a generalised function only existing at one point in time $t=T$, with $T \geq 0$, and defined by

$\int_{t=a}^{b} \delta(t-T) \mathrm{d} t=\left\{\begin{array}{l}1, \text { if } a \leq T \leq b, \\ 0, \text { if } T<a \text { or } T>b .\end{array}\right.$

This function can be looked upon as an infinitely narrow and infinitely tall impulse with a unit area. The Laplace transform of a Dirac function is obtained as the simple exponential function:

$£\{\delta(t-T)\}=\int_{t=0}^{\infty} \delta(t-T) \mathrm{e}^{-s t} \mathrm{~d} t=\mathrm{e}^{-s T}$,

provided that $T \geq 0$.

The NPV theorem

If $x(t)$ is a cash flow, possibly including discrete payments, then the Net Present Value (NPV) of this cash flow is

$N P V=\tilde{x}(\rho)$, 
where $\rho$ is the continuous discount rate, i.e. the Laplace transform of the cash flow, when the frequency $s$ is interpreted as this interest rate $\rho$ [18].

As far as the author knows, there have been no major theoretical developments concerning the Laplace transform of truncated functions, such as regarding a function of the type $f^{+}(t)=\operatorname{Max}(0, f(t))$, which often are present in production-economic problems. For explaining developments below, we therefore include the following relations between a discrete monotonically non-decreasing staircase function $f(t)$ of discrete time and its truncated version $f^{+}(t)=\operatorname{Max}(0, f(t))$ and their respective Laplace transforms.

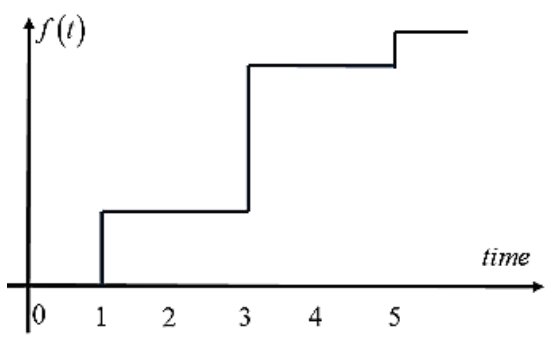

Figure 1 A typical staircase function in discrete time

If $f(t)$ is a staircase function of discrete time (Fig. 1), its time derivative may be written as a train of Dirac impulse functions

$\frac{\mathrm{d} f(t)}{\mathrm{d} t}=f_{0} \delta(t)+\left(f_{1}-f_{2}\right) \delta(t-1)+\left(f_{2}-f_{1}\right) \delta(t-2), \ldots$

where $f_{i}$ is the value of $f(t)$ for $i \leq t<(i+1)$. The transform of $\mathrm{d} f / \mathrm{d} t$ is then by (3)

$$
\begin{aligned}
& £\left\{\frac{\mathrm{d} f(t)}{\mathrm{d} t}\right\}=f_{0}+\left(f_{1}-f_{2}\right) \mathrm{e}^{-s}+\left(f_{2}-f_{1}\right) \mathrm{e}^{-2 s}+\ldots= \\
& =\left(1-\mathrm{e}^{-s}\right) \sum_{i=0}^{\infty} f_{i} \mathrm{e}^{-i s},
\end{aligned}
$$

and the transform of $f(t)$ will be by (4)

$$
\tilde{f}(s)=\frac{1}{s} £\left\{\frac{\mathrm{d} f(t)}{\mathrm{d} t}\right\}=\frac{\left(1-\mathrm{e}^{-s}\right) \sum_{i=0}^{\infty} f_{i} \mathrm{e}^{-i s}}{s} .
$$

If $f(t)$ is monotonically non-decreasing, the truncation $f^{+}(t)=\operatorname{Max}(0, f(t))$ will have the transform

$$
\tilde{f}^{+}(s)=\frac{1}{s} £\{\operatorname{Max}(0, f(t))\}=\frac{\left(1-\mathrm{e}^{-s}\right) \sum_{i=T}^{\infty} f_{i} \mathrm{e}^{-i s}}{s},
$$

where $T$ is the first time that $f(t)$ becomes positive. The transform of the derivative of the truncated function $\mathrm{d} f^{+}(t) / \mathrm{d} t=\mathrm{d} \operatorname{Max}(0, f(t)) / \mathrm{d} t$ will therefore be

$$
s \tilde{f}^{+}(s)=£\{\operatorname{Max}(0, f(t))\}=\left(1-\mathrm{e}^{-s}\right) \sum_{i=T}^{\infty} f_{i} \mathrm{e}^{-i s} .
$$

\section{Impulse trains}

If we have a discrete time function $x(t)$, there is often a need for mathematically describing only the times, $t_{0}, t_{1}$, $t_{2}, \ldots$, when this function has a non-zero value. If the transform of this transform is written $\tilde{x}(s)=\sum_{i=0}^{\infty} x_{i} \mathrm{e}^{-i s t_{i}}$, then its timing is obtained as a train of unit impulses, written

$$
\tilde{x}(s)=\sum_{i=0}^{\infty} x_{i} \mathrm{e}^{-i s t_{i}} .
$$

\section{MRP THEORY WITH AN INITIAL STOCK}

Throughout, we confine ourselves to assembly systems, for which the input matrix may be written as a triangular matrix.

We start with a system with no initial available inventory $\boldsymbol{R}(0)=\mathbf{0}$, where $\boldsymbol{R}(t)$ is the vector of available inventory of each item and $\mathbf{0}$ a column vector of zero-valued components. Disregarding lead times initially, with $\boldsymbol{H}$ as the input matrix describing the Bill-of-Materials (BOM) and $\boldsymbol{P}$ the column vector describing total production of all items, then $\boldsymbol{H P}$ will be the dependent (internal) demand (of sub-items) and $\boldsymbol{P}$ $\boldsymbol{H P}=(\boldsymbol{I}-\boldsymbol{H}) \boldsymbol{P}$ will be net production, which is possible to export from the system. If $\boldsymbol{D}$ is a vector giving the externally demanded quantities of all items, then this demand will be exactly satisfied (neither surplus nor shortage), when $(\boldsymbol{I}-\boldsymbol{H}) \boldsymbol{P}=\boldsymbol{D}$, i.e. when

$$
\boldsymbol{P}=(\boldsymbol{I}-\boldsymbol{H})^{-1} \boldsymbol{D},
$$

where $(\boldsymbol{I}-\boldsymbol{H})^{-1}$ is the Leontief inverse.

Making use of the time translation theorem of Laplace transforms (6), we introduce the diagonal lead-time matrix $\boldsymbol{\tau}(s)$, with $\mathrm{e}^{s \tau_{i}}$ in its $i$ th diagonal position, where $\tau_{i}$ is the lead time for item $i$, i.e. the time ahead of the completion of item $i$ that necessary sub-items for the assembly of this item need to be in place. If $\tilde{\boldsymbol{P}}(s)$ is the transform of (time-varying) production, then this production creates an internal (dependent) demand amounting to $\boldsymbol{H} \tilde{\boldsymbol{\tau}}(s) \tilde{\boldsymbol{P}}(s)$, this expression capturing both the size of the demand of different items and the times at which this demand occurs. Hence, the transform of net production will be $(\boldsymbol{I}-\boldsymbol{H} \tilde{\boldsymbol{\tau}}(s)) \tilde{\boldsymbol{P}}(s)$, so if external demand is to be exactly satisfied (both in size and timing), we must have $(\boldsymbol{I}-\boldsymbol{H} \tilde{\boldsymbol{\tau}}(s)) \tilde{\boldsymbol{P}}(s)=\tilde{\boldsymbol{D}}(s)$, or

$$
\tilde{\boldsymbol{P}}(s)=(\boldsymbol{I}-\boldsymbol{H} \tilde{\boldsymbol{\tau}}(s))^{-1} \tilde{\boldsymbol{D}}(s),
$$

where $\tilde{\boldsymbol{D}}(s)$ is the transform of given (or estimated, forecasted) external demand.

Eq. (17) provides the Lot-for-Lot solution (L4L, "As Required") to all MRP problems, when there is no initial available inventory. Other standard ordering policies in MRP are primarily Fixed Order Quantity (FOQ) and Fixed Period Requirements (FPR), cf. [33].

The L4L policy $\tilde{\boldsymbol{P}}_{\mathrm{L} 4 \mathrm{~L}}(s)$ minimises available inventory throughout the process, so it must be optimal when the costs 
for holding inventory are very high or the ordering costs very low.

In contrast, the All-at-Once policy ( $\forall @ 1)$ minimises the number of setups (when the production of each item is initialised). So each item is then produced in only one batch and this policy is optimal, when the costs for setups are very high (or the holding costs very low). Regarding total amounts, as before, we must have $(\boldsymbol{I}-\boldsymbol{H}) \boldsymbol{P}_{\forall @ 1}=\boldsymbol{D}$, but the timing of production will be different. If the batch for item $i$ is to be completed at time $T_{i}$, then

$$
\tilde{\boldsymbol{P}}_{\forall @ 1}(s)=\left[\begin{array}{ccc}
\mathrm{e}^{-s T_{1}} & 0 & 0 \\
0 & \ddots & 0 \\
0 & 0 & \mathrm{e}^{-s T_{n}}
\end{array}\right] \boldsymbol{P}_{\forall @ 1}=\tilde{\boldsymbol{T}}(s) \boldsymbol{P}_{\forall @ 1},
$$

defining the diagonal matrix $\tilde{\boldsymbol{T}}(s)$, and internal demand will be $\boldsymbol{H} \tilde{\boldsymbol{\tau}}(s) \tilde{\boldsymbol{P}}_{\forall @ 1}(s)$. The times $T_{i}$ are determined sequentially beginning at the top level (an end item having no internal demand) and proceeding downwards. On each level this batch completion time will be determined by the expression

$$
T_{i}=\underset{t}{\arg \min }\left\{\operatorname{Max}\left(0, £^{-1}\left\{[\boldsymbol{H}]_{\mathrm{row} i} \tilde{\boldsymbol{\tau}}(s) \tilde{\overline{\boldsymbol{P}}}_{\forall @ 1}(s)+\tilde{\overline{\boldsymbol{D}}}_{i}(s)\right\}\right)>0\right\},(
$$

where $\tilde{\overline{\boldsymbol{D}}}_{i}(s)=\tilde{\boldsymbol{D}}_{i}(s) / s$ denotes the transform of cumulative demand for item $i$, cf. (4), and which gives $T_{i}$ as the earliest time that there is a positive internal or external demand for this item. The triangular nature of $\boldsymbol{H}$ ensures that for each batch, only times previously determined on higher levels enter when searching for the current earliest completion time.

Concerning economic consequences from choosing different production policies, we introduce three unit price and cost vectors (row vectors), namely $\boldsymbol{p}$ for sales prices and $c$ for unit variable costs of the items, and $K$ as the setup cost vector, capturing the fixed costs associated with producing/purchasing a batch of the respective items. Concerning $\boldsymbol{K}$, these costs are allocated to the completion time of the respective batches. Instead, if they were referring to the starting times of batches, we can exchange $\boldsymbol{K}$ for an adjusted setup cost vector $\boldsymbol{K} \tilde{\boldsymbol{\tau}}(s)$ moving the associated payments backwards in time by the respective lead times. Options for in-between timing are also easily taken care of.

With these basic payment parameters, we interpret $\boldsymbol{p} \tilde{\boldsymbol{D}}(s)$ to be the transform of revenues, $\boldsymbol{c} \tilde{\boldsymbol{P}}(s)$ the transform of variable production costs and $\boldsymbol{K} \tilde{\boldsymbol{P}}^{\prime}(s)$ to be the transform of out-payments for setups, where $\tilde{\boldsymbol{P}}^{\prime}(s)$ is the train of impulses for the completion times of batches, cf. (15). For the All-at-Once policy $\tilde{\boldsymbol{P}}_{\forall @ 1}^{\prime}(s)$ will coincide with $\tilde{\boldsymbol{T}}_{\forall @ 1}(s) \boldsymbol{1}$, where $\mathbf{1}$ is a column vector of unit components. Applying the $N P V$ theorem (9), the Net Present Value (NPV) collecting all modelled economic consequences into one financial measure is obtained as

$$
N P V=\boldsymbol{p} \tilde{\boldsymbol{D}}(\rho)-c \tilde{\boldsymbol{P}}(\rho)-\boldsymbol{K} \tilde{\boldsymbol{P}}^{\prime}(\rho),
$$

where the frequency $s$ has been exchanged for the continuous discount rate $\rho$ in the transforms. This equation presupposes that all inventory holding costs are capital costs, or modified capital costs (by raising the discount rate $\rho$ appropriately in order to cover physical holding costs such as rent, manual holding, insurance, refrigeration, etc.). If out-of-pocket costs need to be taken care of more accurately, the equation needs to be modified accordingly, see [32].

Holding costs are usually referred to inventory. The coarser method of attaching holding costs to the physical level of inventory may here be interpreted as the difference between the undiscounted and discounted cash flow, to which the (undiscounted) setup costs are added, making up the inventory-related costs, i.e.

$$
\begin{aligned}
& I R C=\boldsymbol{p} \tilde{\boldsymbol{D}}(0)-\boldsymbol{c} \tilde{\boldsymbol{P}}(0)-\boldsymbol{K} \tilde{\boldsymbol{P}}^{\prime}(0)- \\
& -\left(\boldsymbol{p} \tilde{\boldsymbol{D}}(\rho)-\boldsymbol{c} \tilde{\boldsymbol{P}}(\rho)-\boldsymbol{K} \tilde{\boldsymbol{P}}^{\prime}(\rho)\right)+\boldsymbol{K} \tilde{\boldsymbol{P}}^{\prime}(0)= \\
& =\boldsymbol{p}(\tilde{\boldsymbol{D}}(0)-\tilde{\boldsymbol{D}}(\rho))-\boldsymbol{c}(\tilde{\boldsymbol{P}}(0)-\boldsymbol{c} \tilde{\boldsymbol{P}}(\rho))+\boldsymbol{K} \tilde{\boldsymbol{P}}^{\prime}(\rho),
\end{aligned}
$$

where $I R C$ denotes the inventory-related costs, i.e. the sum of capital-holding costs and setup costs. Both measures must give the same result, since $N P V(\rho)+\operatorname{IRC}(\rho)=$ $=\boldsymbol{p} \tilde{\boldsymbol{D}}(0)-\boldsymbol{c} \tilde{\boldsymbol{P}}(0)$, which is a constant (independent of discount rate and setup costs), also illustrated in Section 5.

We now turn to the main purpose of this paper, namely to study modifications to the above results, when there is a non-zero initial inventory of items $\boldsymbol{R}(0)$ available.

Irrespective of whichever production policy $\tilde{\boldsymbol{P}}(s)$ is chosen, available inventory $\boldsymbol{R}$ always follows

$$
\tilde{\boldsymbol{R}}(s)=\frac{\boldsymbol{R}(0)+(\boldsymbol{I}-\boldsymbol{H} \tilde{\boldsymbol{\tau}}(s)) \tilde{\boldsymbol{P}}(s)-\tilde{\boldsymbol{D}}(s)}{s},
$$

in which the time integral theorem of the Laplace transform (4) has been used. When there still are items in stock, it is assumed that they in the first place cover external and internal demand. When these items are used up, in general, there will be a remaining demand, which we denote $\boldsymbol{d}(t)$. In the time domain, $\overline{\boldsymbol{d}}_{i}(t)$ is determined from

$$
\begin{aligned}
& \overline{\boldsymbol{d}}_{i}(t)=\operatorname{Max}\left(0, \overline{\boldsymbol{D}}_{i}(t)+£^{-1}\left\{\sum_{j} \boldsymbol{H}_{i j} \mathrm{e}^{s \tau} \tilde{\overline{\boldsymbol{P}}}_{j}(s)\right\}-\boldsymbol{R}_{i}(0)\right)= \\
& =\operatorname{Max}\left(0, \overline{\boldsymbol{D}}_{i}(t)+\sum_{j} \boldsymbol{H}_{i j} \overline{\boldsymbol{P}}_{j}\left(t+\tau_{j}\right)-\boldsymbol{R}_{i}(0)\right),
\end{aligned}
$$

i.e. where $\overline{\boldsymbol{d}}_{i}(t)$ is the remaining cumulative demand of item $i . \boldsymbol{R}(t)$ is a staircase function. $\overline{\boldsymbol{d}}_{i}(t)$, in general, will depend on the production of items $j^{\prime}$, for $j<i$. If a production plan $\tilde{\boldsymbol{P}}(s)$ is feasible, we must have $\boldsymbol{R}(t) \geq \boldsymbol{0}$, otherwise there will be shortages of components, making the plan impossible to follow. 
The time at which initial inventory is used up, in general, will be different for different items, and is written $T_{i}$ for the $i^{\text {th }}$ item. It is the earliest time at which $\overline{\boldsymbol{d}}_{i}(t)$ is positive:

$$
T_{i}=\underset{t}{\arg \min }\left\{\operatorname{Max}\left(0, \overline{\boldsymbol{d}}_{i}(t)\right)>0\right\} .
$$

For the $\forall @ 1$ policy it will be completion time of the (only) batch of this item. Determining these $T_{i}$ can take place in the same way as explained above beginning with the top level, where there is no internal demand and proceeding downwards, using the times calculated earlier for higher levels.

Once the remaining demand has been determined, the L4L and $\forall @ 1$ policies according to the equations for the initially empty system, will be valid. For the L4L and $\forall @ 1$ policies, we have

$$
\left\{\begin{array}{l}
\tilde{\boldsymbol{P}}_{\mathrm{L} 4 \mathrm{~L}}(s)=\tilde{\boldsymbol{d}}(s), \\
\tilde{\boldsymbol{P}}_{\forall @ 1}(s)=\tilde{\boldsymbol{T}}(s) \tilde{\boldsymbol{d}}(0),
\end{array}\right.
$$

where $\tilde{\boldsymbol{d}}(0)$ contains the total remaining demand for each item, which can be seen from applying (4)-(5), $\overline{\boldsymbol{d}}(\infty)=\frac{\lim _{s \rightarrow 0} s \tilde{\boldsymbol{d}}(s)}{s}=\tilde{\boldsymbol{d}}(0)$.

Applying the final value theorem of Laplace transforms (5) to (22), we find for the final remaining inventory $\boldsymbol{R}(\infty)$ in the $\mathrm{L} 4 \mathrm{~L}$ case

$$
\boldsymbol{R}(\infty)=\lim _{s \rightarrow 0} s \tilde{\boldsymbol{R}}(s)=\boldsymbol{R}(0)+(\boldsymbol{I}-\boldsymbol{H}) \tilde{\boldsymbol{d}}(0)-\tilde{\boldsymbol{D}}(0),
$$

and in the $\forall @ 1$ case, the (expected) same result holds, due to both $\tilde{\boldsymbol{\tau}}(s)$ and $\tilde{\boldsymbol{T}}(s)$ collapsing into the identity matrix $\boldsymbol{I}$ for $s=0$. Eqs (26) may be interpreted as the remaining inventory $\boldsymbol{R}(\infty)$ is initial inventory $\boldsymbol{R}(0)$ subtracted by (i) subitems used up in production $\boldsymbol{H} \tilde{\boldsymbol{d}}(0)$, and (ii), by $(\tilde{\boldsymbol{D}}(0)-\tilde{\boldsymbol{d}}(0))$, which is total demand less total remaining demand (when initial inventory is used up). The case that there is a positive remaining inventory for some item, thus occurs when initial inventory is more than enough to cover external and internal demand for this item.

A simple numerical example explaining these relationships is given in Section 5 below.

\section{ECONOMIC VALUE OF INITIAL STOCK}

We investigate the economic consequences from having, or not having an initial available inventory $\boldsymbol{R}(0)$. Thus, we compare the Net Present Value obtained either we have this initial stock or not. The difference will depend on which production policy is followed.

Starting with an empty inventory, the $N P V$ according to (9) following the L4L policy will be:

$$
\begin{aligned}
& N P V_{\text {empty } \mathrm{L} 4 \mathrm{~L}}=\boldsymbol{p} \tilde{\boldsymbol{D}}(\rho)-\boldsymbol{c} \tilde{\boldsymbol{P}}_{\mathrm{L} 4 \mathrm{~L}, \mathrm{empty}}(\rho)-\boldsymbol{K} \tilde{\boldsymbol{P}}_{\mathrm{L} 4 \mathrm{~L}, \text { empty }}^{\prime}(\rho)= \\
& =\left(\boldsymbol{p}-\boldsymbol{c}(\boldsymbol{I}-\boldsymbol{H} \tilde{\boldsymbol{\tau}}(\rho))^{-1}\right) \tilde{\boldsymbol{D}}(\rho)-\boldsymbol{K} \tilde{\boldsymbol{P}}_{\mathrm{L} 4 \mathrm{~L}, \text { empty }}^{\prime}(\rho),
\end{aligned}
$$

where $\tilde{\boldsymbol{P}}_{\mathrm{L} 4 \mathrm{~L} \text {, empty }}^{\prime}$ the impulse train of production timing associated with $(\boldsymbol{I}-\boldsymbol{H} \tilde{\boldsymbol{\tau}}(\rho))^{-1} \tilde{\boldsymbol{D}}(\rho)$ in this L4L case, cf. (15).

Instead, having an initial stock $\boldsymbol{R}(0)$ will give the $N P V$ :

$$
\begin{aligned}
& N P V_{\mathrm{L} 4 \mathrm{~L}}=\boldsymbol{p} \tilde{\boldsymbol{D}}(\rho)-\boldsymbol{c} \tilde{\boldsymbol{P}}_{\mathrm{L} 4 \mathrm{~L}}(\rho)-\boldsymbol{K} \tilde{\boldsymbol{P}}_{\mathrm{L} 4 \mathrm{~L}}^{\prime}(\rho)= \\
& =\boldsymbol{p} \tilde{\boldsymbol{D}}(\rho)-\boldsymbol{c} \tilde{\boldsymbol{d}}(\rho)-\boldsymbol{K} \tilde{\boldsymbol{d}}^{\prime}(\rho) .
\end{aligned}
$$

So the value of the initial stock in the $\mathrm{L} 4 \mathrm{~L}$ case is

$$
\begin{aligned}
& \triangle N P V_{\mathrm{L} 4 \mathrm{~L}}=N P V_{\mathrm{L} 4 \mathrm{~L}}-N P V_{\text {empty, L4L }}= \\
& =\boldsymbol{c}\left((\boldsymbol{I}-\boldsymbol{H} \tilde{\boldsymbol{\tau}}(\rho))^{-1} \tilde{\boldsymbol{D}}(\rho)-\tilde{\boldsymbol{d}}(\rho)\right)+\boldsymbol{K}\left(\tilde{\boldsymbol{P}}_{\mathrm{L} 4 \mathrm{~L}, \text { empty }}^{\prime}(\rho)-\tilde{\boldsymbol{d}}^{\prime}(\rho)\right) .
\end{aligned}
$$

This expression presupposes that (i) that the given demand can be satisfied when $\boldsymbol{R}(0)=\boldsymbol{0}$, and (ii) that if there might be a remaining positive, available inventory $\boldsymbol{R}(\infty)$, the value of this inventory is worthless.

In the $\forall @ 1$ case, we instead obtain

$$
\begin{aligned}
& N P V_{\text {empty }, \forall @ 1}= \\
& =\boldsymbol{p} \tilde{\boldsymbol{D}}(\rho)-\boldsymbol{c} \tilde{\boldsymbol{P}}_{\forall @ 1, \text { empty }}(\rho)-\boldsymbol{K} \tilde{\boldsymbol{P}}_{\forall @ 1, \text { empty }}^{\prime}(\rho)= \\
& =\boldsymbol{p} \tilde{\boldsymbol{D}}(\rho)-\boldsymbol{c} \tilde{\boldsymbol{T}}_{\text {empty }}(\rho) \tilde{\boldsymbol{D}}(0)-\boldsymbol{K} \tilde{\boldsymbol{T}}_{\text {empty }}(\rho) \boldsymbol{1},
\end{aligned}
$$

where $\tilde{\boldsymbol{T}}_{\text {empty }}$ is the matrix containing diagonal elements $\mathrm{e}^{-s T_{i}}$ with $T_{i}$ being the earliest time that item $i$ is demanded internally or externally, and:

$$
\begin{aligned}
& N P V_{\forall @ 1}=\boldsymbol{p} \tilde{\boldsymbol{D}}(\rho)-\boldsymbol{c} \tilde{\boldsymbol{P}}_{\forall @ 1}(\rho)-\boldsymbol{K} \tilde{\boldsymbol{P}}_{\forall @ 1}^{\prime}(\rho)= \\
& =\boldsymbol{p} \tilde{\boldsymbol{D}}(\rho)-\boldsymbol{c} \tilde{\boldsymbol{T}}(\rho) \tilde{\boldsymbol{d}}(0)-\boldsymbol{K} \tilde{\boldsymbol{T}}(\rho) \boldsymbol{1} .
\end{aligned}
$$

Therefore the value of initial inventory will be:

$$
\begin{aligned}
& \triangle N P V_{\forall @ 1}=N P V_{\forall @ 1}-N P V_{\text {empty }, \forall @ 1}= \\
& =\boldsymbol{c}\left(\tilde{\boldsymbol{T}}_{\text {empty }}(\rho) \tilde{\boldsymbol{D}}(0)-\tilde{\boldsymbol{T}}(\rho) \tilde{\boldsymbol{d}}(0)\right)+\boldsymbol{K}\left(\tilde{\boldsymbol{T}}_{\text {empty }}(\rho)-\tilde{\boldsymbol{T}}(\rho)\right) \boldsymbol{1} .
\end{aligned}
$$

The same qualification applies to this evaluation as in the L4L case.

It should be pointed out that the timing vector $\tilde{\boldsymbol{T}}_{\forall @ 1}(\rho) \boldsymbol{1}$ always coincides with the timing vector $\tilde{\boldsymbol{P}}_{\forall @ 1}^{\prime}(\rho)$.

\section{A NUMERICAL EXAMPLE}

Let us consider a product A made up of sub-items as illustrated by the product structure tree in Fig. 2. For producing one item $\mathrm{A}$, one sub-item $\mathrm{B}$ and two items $\mathrm{C}$ are required ahead of the completion of $A$ by 1 time unit (the lead time of A). Item B, in its turn, is made from one unit of a fourth item $\mathrm{D}$ required two time units in advance of the 
completion of B. Numbering the items alphabetically, the Bill-of-Materials is thus given by the input matrix $\boldsymbol{H}$, and the lead times are captured in the lead time matrix $\tilde{\boldsymbol{\tau}}(s)$ :

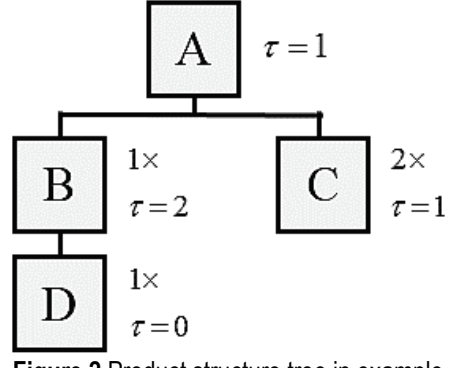

$\boldsymbol{H}=\left[\begin{array}{cccc}0 & 0 & 0 & 0 \\ 1 & 0 & 0 & 0 \\ 2 & 0 & 0 & 0 \\ 0 & 1 & 0 & 0\end{array}\right], \tilde{\boldsymbol{\tau}}(s)=\left[\begin{array}{cccc}\mathrm{e}^{s} & 0 & 0 & 0 \\ 0 & \mathrm{e}^{2 s} & 0 & 0 \\ 0 & 0 & \mathrm{e}^{s} & 0 \\ 0 & 0 & 0 & 1\end{array}\right]$. inverse

So this enables us to determine the generalised Leontief $(\boldsymbol{I}-\boldsymbol{H} \tilde{\boldsymbol{\tau}}(s))^{-1}=\left[\begin{array}{cccc}1 & 0 & 0 & 0 \\ \mathrm{e}^{s} & 1 & 0 & 0 \\ 2 \mathrm{e}^{s} & 0 & 1 & 0 \\ \mathrm{e}^{3 s} & \mathrm{e}^{2 s} & 0 & 1\end{array}\right], \boldsymbol{R}(0)=\left[\begin{array}{l}5 \\ 1 \\ 2 \\ 2\end{array}\right]$.

Let us consider a time span of six time units (including a current period 0 ) and that external demand is present only for the end product $\mathrm{A}$ and is given by discrete period demand $\boldsymbol{D}_{1}(t)$ (using the notation $D_{t}=\boldsymbol{D}_{1}(t)$ ) according to

$$
\boldsymbol{D}_{1}(t)=\left[D_{0}, D_{1}, D_{2}, D_{3}, D_{4}, D_{5}\right]=[0,2,1,3,1,2] .
$$

Cumulative demand $\overline{\boldsymbol{D}}_{1}(t)$ will then be:

$$
\overline{\boldsymbol{D}}_{1}(t)=\left[\begin{array}{l}
D_{0}, D_{0}+D_{1}, D_{0}+D_{1}+D_{2}, \\
D_{0}+D_{1}+D_{2}+D_{3}, \\
D_{0}+D_{1}+D_{2}+D_{3}+D_{4}, \\
D_{0}+D_{1}+D_{2}+D_{3}+D_{4}+D_{5}
\end{array}\right]=[0,2,3,6,7,9]
$$

The Laplace transform of the (external) demand for this end item will be $\tilde{\boldsymbol{D}}_{1}(s)=2 \mathrm{e}^{-s}+\mathrm{e}^{-2 s}+3 \mathrm{e}^{-3 s}+\mathrm{e}^{-4 s}+2 \mathrm{e}^{-5 s}$. The external demand and the cumulative external demand vectors are thus

$$
\begin{aligned}
& \tilde{\boldsymbol{D}}(s)=\left[\begin{array}{l}
2 \mathrm{e}^{-s}+\mathrm{e}^{-2 s}+3 \mathrm{e}^{-3 s}+\mathrm{e}^{-4 s}+2 \mathrm{e}^{-5 s} \\
0 \\
0 \\
0
\end{array}\right], \\
& \tilde{\overline{\boldsymbol{D}}}(s)=\frac{1}{s}\left[\begin{array}{l}
2 \mathrm{e}^{-s}+\mathrm{e}^{-2 s}+3 \mathrm{e}^{-3 s}+\mathrm{e}^{-4 s}+2 \mathrm{e}^{-5 s} \\
0 \\
0
\end{array}\right] .
\end{aligned}
$$

Depending on production policy $\tilde{\boldsymbol{P}}(s)$, as stated by (21), available inventory will develop according to

$$
\begin{aligned}
& \tilde{\boldsymbol{R}}(s)=\frac{1}{s}\left[\begin{array}{l}
5 \\
1 \\
2 \\
2
\end{array}\right]+\frac{1}{s}\left(\left[\begin{array}{llll}
1 & 0 & 0 & 0 \\
0 & 1 & 0 & 0 \\
0 & 0 & 1 & 0 \\
0 & 0 & 0 & 1
\end{array}\right]-\left[\begin{array}{cccc}
0 & 0 & 0 & 0 \\
\mathrm{e}^{s} & 0 & 0 & 0 \\
2 \mathrm{e}^{s} & 0 & 0 & 0 \\
0 & \mathrm{e}^{2 s} & 0 & 0
\end{array}\right]\right)\left[\begin{array}{c}
\tilde{\boldsymbol{P}}_{1}(s) \\
\tilde{\boldsymbol{P}}_{2}(s) \\
\tilde{\boldsymbol{P}}_{3}(s) \\
\tilde{\boldsymbol{P}}_{4}(s)
\end{array}\right]- \\
& -\frac{1}{s}\left[\begin{array}{l}
2 \mathrm{e}^{-s}+\mathrm{e}^{-2 s}+3 \mathrm{e}^{-3 s}+\mathrm{e}^{-4 s}+2 \mathrm{e}^{-5 s} \\
0 \\
0 \\
0
\end{array}\right],
\end{aligned}
$$

the first term showing the assumed initial inventory $\boldsymbol{R}(0) / s$, cf. (22).

Starting with the top level, we now proceed to find the earliest time $T_{1}$ when there is a positive external or internal demand (above initial inventory). For A (item 1), we have $\tilde{\boldsymbol{R}}_{1}(s)=\tilde{\overline{\boldsymbol{P}}}_{1}(s)+\left(5-\left(2 \mathrm{e}^{-s}+\mathrm{e}^{-2 s}+3 \mathrm{e}^{-3 s}+\mathrm{e}^{-5 s}\right)\right)$, which shows that initial inventory does not cover cumulative demand for more than periods $0-2$, so for this item $T_{1}=3$, i.e. $\boldsymbol{P}_{1}(t)$ needs to be positive at $t=3$ for either policy. Looking within the time domain, using (38) we have $\left(\overline{\boldsymbol{D}}_{1}\left(T_{1}\right)-5\right)^{+}=$ $\operatorname{Max}\left(0, \overline{\boldsymbol{D}}_{1}\left(T_{1}\right)-5\right)=[0,0,0,1,1,2]$, also showing that $T_{1}$ $=3$ both for L4L and $\forall @ 1$.

On level 2 (choosing the B item) there is only internal demand. From (21) we read $\tilde{\boldsymbol{R}}_{2}(s)=\left(1+\tilde{\boldsymbol{P}}_{2}(s)-\mathrm{e}^{s} \tilde{\boldsymbol{P}}_{1}(s)\right) / s$, which shows that $\overline{\boldsymbol{P}}_{2}(t)$ must cover $\overline{\boldsymbol{P}}_{1}(t+1)-\tilde{\boldsymbol{R}}_{2}(0)=$ $\overline{\boldsymbol{P}}_{1}(t+1)-1$, so the first time that $\overline{\boldsymbol{P}}_{1}(t+1)>1$, requires $\boldsymbol{P}_{2}(t)$ $>0$ and $T_{2}=t$.

For the third item we have $\tilde{\boldsymbol{R}}_{3}(s)=$ $\left(2+\tilde{\boldsymbol{P}}_{3}(s)-2 e^{s} \tilde{\boldsymbol{P}}_{1}(s)\right) / s, \quad$ or $\quad \boldsymbol{R}_{3}(t)=\left(2+\overline{\boldsymbol{P}}_{3}(s)-2 \overline{\boldsymbol{P}}_{1}(t+1)\right)$, which similarly shows that if $\overline{\boldsymbol{P}}_{1}(t+1)>1$, then $\boldsymbol{P}_{3}(t)>0$ and $T_{3}=t$. For our final item 4 (D), we have $\tilde{\boldsymbol{R}}_{4}(s)=$ $\left(3+\tilde{\boldsymbol{P}}_{4}(s)-\mathrm{e}^{2 s} \tilde{\boldsymbol{P}}_{2}(s)\right) / s$, or $\boldsymbol{R}_{4}(t)=3+\overline{\boldsymbol{P}}_{4}(t)-\overline{\boldsymbol{P}}_{2}(t+2)$, so the first time that $\overline{\boldsymbol{P}}_{2}(t+2)>3$ necessitates $\overline{\boldsymbol{P}}_{4}(t)$ to be 
positive, i.e. $T_{4}=t-2$. The time development for internal and external demand and production for the four items following the two policies are illustrated in Figs. 3-6.

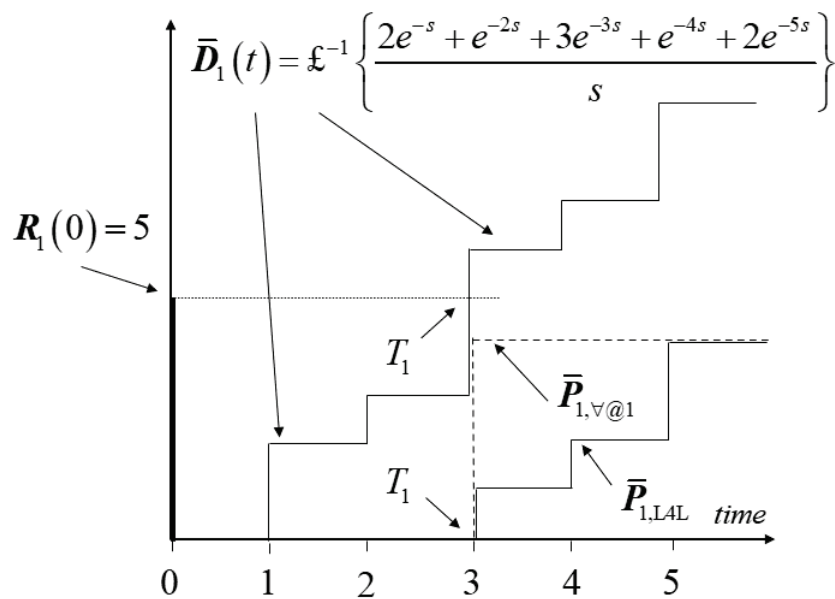

Figure 3 External demand of end item $A$ as a staircase function and production satisfying this demand, L4L solid and $\forall @ 1$ dashed.

On the top level with no internal demand, we have $\overline{\boldsymbol{d}}_{1}(t)=\operatorname{Max}\left(0, \overline{\boldsymbol{D}}_{1}(t)-\boldsymbol{R}_{1}(0)\right)=\operatorname{Max}\left(0, \overline{\boldsymbol{D}}_{1}\left(T_{1}\right)-5\right)=$ $[0,0,0,1,1,2]$. So the transform of cumulate remaining demand becomes $\quad \tilde{\bar{d}}_{1}(s)=\left(\mathrm{e}^{-3 s}+\mathrm{e}^{-4 s}+2 \mathrm{e}^{-5 s}\right) / s$, and of remaining demand $\tilde{\boldsymbol{d}}_{1}(s)=\left(\mathrm{e}^{-3 s}+\mathrm{e}^{-4 s}+2 \mathrm{e}^{-5 s}\right)$. With the L4L policy $\quad \tilde{\boldsymbol{P}}_{1, \mathrm{~L} 4 \mathrm{~L}}(s)=\tilde{\boldsymbol{d}}_{1}(s)$, and with $\forall @ 1$, $\tilde{\boldsymbol{P}}_{1, \forall @ 1}(s)=\mathrm{e}^{-3 s} \tilde{\boldsymbol{d}}_{1}(0)=4 \mathrm{e}^{-3 s}$. Thus $T_{1}=3$. To summarise,

$$
\left\{\begin{array}{l}
\tilde{\boldsymbol{P}}_{1, \mathrm{~L} 4 \mathrm{~L}}(s)=\left(\mathrm{e}^{-3 s}+\mathrm{e}^{-4 s}+2 \mathrm{e}^{-5 s}\right), \\
\tilde{\boldsymbol{P}}_{1, \forall @ 1}(s)=4 \mathrm{e}^{-3 s}, \\
T_{1, \mathrm{~L} 4 \mathrm{~L}}=T_{1, \forall @ 1}=3 .
\end{array}\right.
$$

For item 2 (B), and the L4L policy, we have $\overline{\boldsymbol{d}}_{2}(t)=\operatorname{Max}\left(0, \overline{\boldsymbol{P}}_{1, \mathrm{~L} 4 \mathrm{~L}}(t+1)-\boldsymbol{R}_{2}(0)\right), \quad$ or $\quad \tilde{\overline{\boldsymbol{d}}}_{2}(s)=$ $=£\left\{\operatorname{Max}\left(0, £^{-1}\left\{\left(\mathrm{e}^{s} \tilde{\boldsymbol{P}}_{1, \mathrm{~L} 4 \mathrm{~L}}(s)-\boldsymbol{R}_{2}(0)\right) / s\right\}\right)\right\}=$ $=£\left\{\operatorname{Max}\left(0, \AA^{-1}\left\{\left(\mathrm{e}^{s}\left(\mathrm{e}^{-3 s}+\mathrm{e}^{-4 s}+2 \mathrm{e}^{-5 s}\right)-1\right) / s\right\}\right)\right\}=$ $=\left(\mathrm{e}^{-3 s}+2 \mathrm{e}^{-4 s}\right) / s \quad$ and $\quad \tilde{\boldsymbol{d}}_{2}(s)=\left(\mathrm{e}^{-3 s}+2 \mathrm{e}^{-4 s}\right), \quad$ so $\tilde{\boldsymbol{P}}_{2, \mathrm{~L} 4 \mathrm{~L}}(s)=\tilde{\boldsymbol{d}}_{2}(s)=\left(\mathrm{e}^{-3 s}+2 \mathrm{e}^{-4 s}\right)$ giving $T_{2}=3$. And for the $\forall @ 1$ policy, we obtain $\tilde{\boldsymbol{P}}_{2, \forall @ 1}(s)=\mathrm{e}^{-T_{2} s} \tilde{\boldsymbol{d}}_{2}(0)=3 \mathrm{e}^{-2 s}$.

Summarising, we have:

$$
\left\{\begin{array}{l}
\tilde{\boldsymbol{P}}_{2, \mathrm{~L} 4 \mathrm{~L}}(s)=\mathrm{e}^{-3 s}+2 \mathrm{e}^{-4 s}, \\
T_{2, \mathrm{~L} 4 \mathrm{~L}}=3, \\
\tilde{\boldsymbol{P}}_{2, \forall @ 1}(s)=3 \mathrm{e}^{-2 s}, \\
T_{2, \forall @ 1}=2 .
\end{array}\right.
$$

Similarly, for Item $\mathrm{C}$ and L4L, we have $\overline{\boldsymbol{d}}_{3}(t)=\operatorname{Max}\left(0,2 \overline{\boldsymbol{P}}_{1, \mathrm{~L} 4 \mathrm{~L}}(t+1)-\boldsymbol{R}_{3}(0)\right), \quad$ or $\quad \tilde{\overline{\boldsymbol{d}}}_{3}(s)=$ $=£\left\{\operatorname{Max}\left(0, £^{-1}\left\{\left(2 \mathrm{e}^{s} \tilde{\boldsymbol{P}}_{1, \mathrm{~L} 4 \mathrm{~L}}(s)-\boldsymbol{R}_{3}(0)\right) / s\right\}\right)\right\}=$ $=£\left\{\operatorname{Max}\left(0, £^{-1}\left\{2\left(\mathrm{e}^{-2 s}+\mathrm{e}^{-3 s}+2 \mathrm{e}^{-4 s}-1\right) / s\right\}\right)\right\}=$ $=\left(2 \mathrm{e}^{-3 s}+4 \mathrm{e}^{-4 s}\right) / s$. So $\tilde{\boldsymbol{P}}_{3, \mathrm{~L} 4 \mathrm{~L}}(s)=\tilde{\boldsymbol{d}}_{3}(s)=\left(2 \mathrm{e}^{-3 s}+4 \mathrm{e}^{-4 s}\right)$ and $T_{3}=3$. Instead for $\forall @ 1, T_{3}=2$ and we obtain $\tilde{\boldsymbol{P}}_{3, \forall @ 1}(s)=\mathrm{e}^{-s T_{3}} \tilde{\boldsymbol{d}}_{3}(0)=6 \mathrm{e}^{-2 s}$. So, summarising:

$$
\left\{\begin{array}{l}
\tilde{\boldsymbol{P}}_{3, \mathrm{~L} 4 \mathrm{~L}}(s)=\left(2 \mathrm{e}^{-3 s}+4 \mathrm{e}^{-4 s}\right), \\
T_{3, \mathrm{~L} 4 \mathrm{~L}}=3, \\
\tilde{\boldsymbol{P}}_{3, \forall @ 1}(s)=6 \mathrm{e}^{-2 s}, \\
T_{3, \forall @ 1}=2 .
\end{array}\right.
$$

Finally for item 4 (D in Fig. 2), which has an initial available inventory of $\boldsymbol{R}_{4}(0)=2$, we have $\overline{\boldsymbol{d}}_{4}(t)=$ $=£\left\{\operatorname{Max}\left(0, £^{-1}\left\{\frac{\left(\mathrm{e}^{2 s}\left(\mathrm{e}^{-3 s}+2 \mathrm{e}^{-4 s}\right)-2\right)}{s}\right\}\right)\right\}$, and therefore in the L4L case $\tilde{\overline{\boldsymbol{d}}}_{4}(s)=$ $=£\left\{\operatorname{Max}\left(0, £^{-1}\left\{\left(\mathrm{e}^{2 s} \tilde{\boldsymbol{P}}_{2, \mathrm{~L} 4 \mathrm{~L}}(s)-\boldsymbol{R}_{4}(0)\right) / s\right\}\right)\right\}=$ $=£\left\{\operatorname{Max}\left(0, £^{-1}\left\{\left(\left(\mathrm{e}^{-s}+2 \mathrm{e}^{-2 s}\right)-2\right) / s\right\}\right)\right\}=\mathrm{e}^{-2 s} / s, \quad$ so $T_{4}=2$, and according to the $\forall @ 1$ policy $T_{4}=0$, so summing up

$$
\left\{\begin{array}{l}
\tilde{\boldsymbol{P}}_{4, \mathrm{~L} 4 \mathrm{~L}}(s)=\mathrm{e}^{-2 s}, \\
T_{4, \mathrm{~L} 4 \mathrm{~L}}=2, \\
\tilde{\boldsymbol{P}}_{4, \forall @ 11}(s)=1, \\
T_{4, \forall @ 1}=0 .
\end{array}\right.
$$

Assembling the components from (39)-(42), the production vectors and $\tilde{\boldsymbol{T}}(s)$ matrices will be: 


$$
\begin{aligned}
& \tilde{\boldsymbol{P}}_{\mathrm{L} 4 \mathrm{~L}}(s)=\left[\begin{array}{l}
\mathrm{e}^{-3 s}+\mathrm{e}^{-4 s}+2 \mathrm{e}^{-5 s} \\
\mathrm{e}^{-3 s}+2 \mathrm{e}^{-4 s} \\
2 \mathrm{e}^{-3 s}+4 \mathrm{e}^{-4 s} \\
\mathrm{e}^{-2 s}
\end{array}\right], \tilde{\boldsymbol{P}}_{\forall @ 1}(s)=\left[\begin{array}{l}
4 \mathrm{e}^{-3 s} \\
3 \mathrm{e}^{-2 s} \\
6 \mathrm{e}^{-2 s} \\
1
\end{array}\right], \\
& \tilde{\boldsymbol{T}}_{\mathrm{L} 4 \mathrm{~L}}(s)=\left[\begin{array}{cccc}
\mathrm{e}^{-3 s} & 0 & 0 & 0 \\
0 & \mathrm{e}^{-3 s} & 0 & 0 \\
0 & 0 & \mathrm{e}^{-3 s} & 0 \\
0 & 0 & 0 & \mathrm{e}^{-2 s}
\end{array}\right], \tilde{\boldsymbol{T}}_{\forall @ 1}(s)=\left[\begin{array}{cccc}
\mathrm{e}^{-3 s} & 0 & 0 & 0 \\
0 & \mathrm{e}^{-2 s} & 0 & 0 \\
0 & 0 & \mathrm{e}^{-2 s} & 0 \\
0 & 0 & 0 & 1
\end{array}\right] .(4
\end{aligned}
$$

In addition, from (43), we find the setup timing vectors of the two policies $\tilde{\boldsymbol{P}}_{\mathrm{L} 4 \mathrm{~L}}^{\prime}(s), \tilde{\boldsymbol{P}}_{\forall @ 1}^{\prime}(s)=\boldsymbol{T}_{\forall @ 1}(s) \boldsymbol{1}$ to be

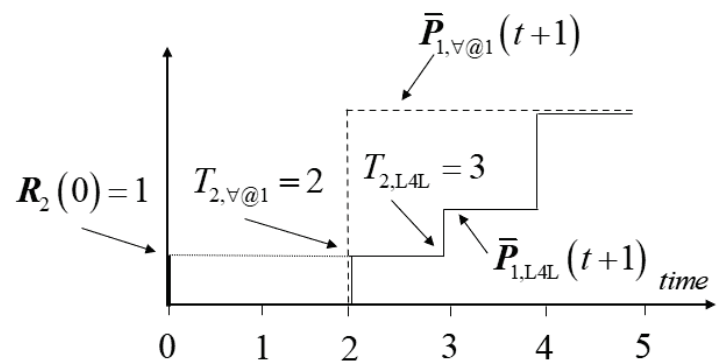

Figure 4 Item $B$ developments, to the left internally generated demand (one time unit earlier), and to the right production satisfying this demand, L4L solid and $\forall @ 1$ dashed.
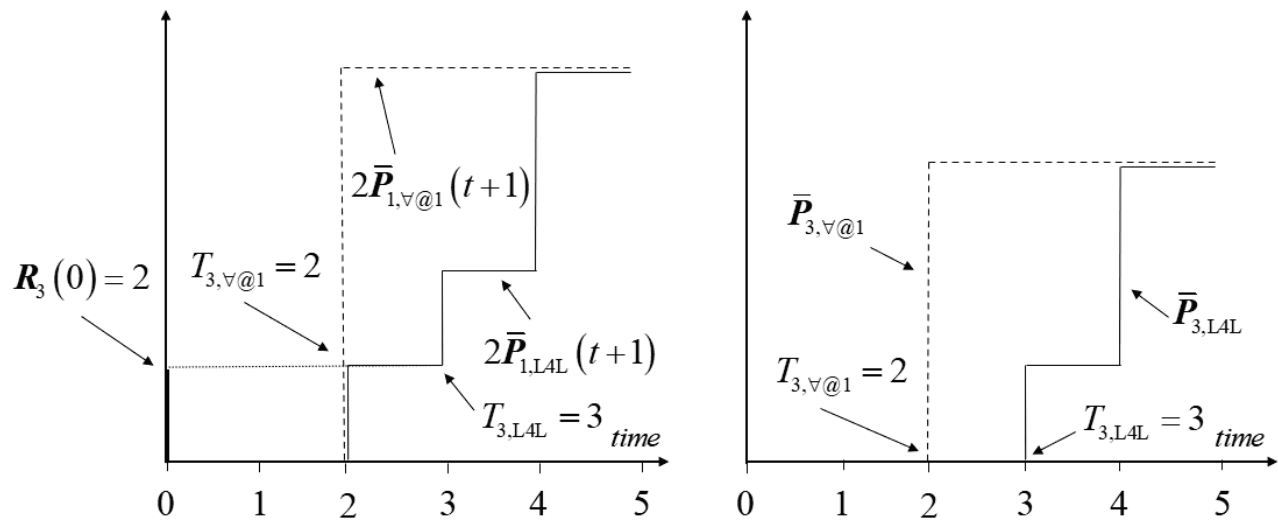

Figure 5 Item $C$ developments, to the left internal demand, generated one time unit earlier, and to the right production, L4L solid and $\forall @ 1$ dashed.

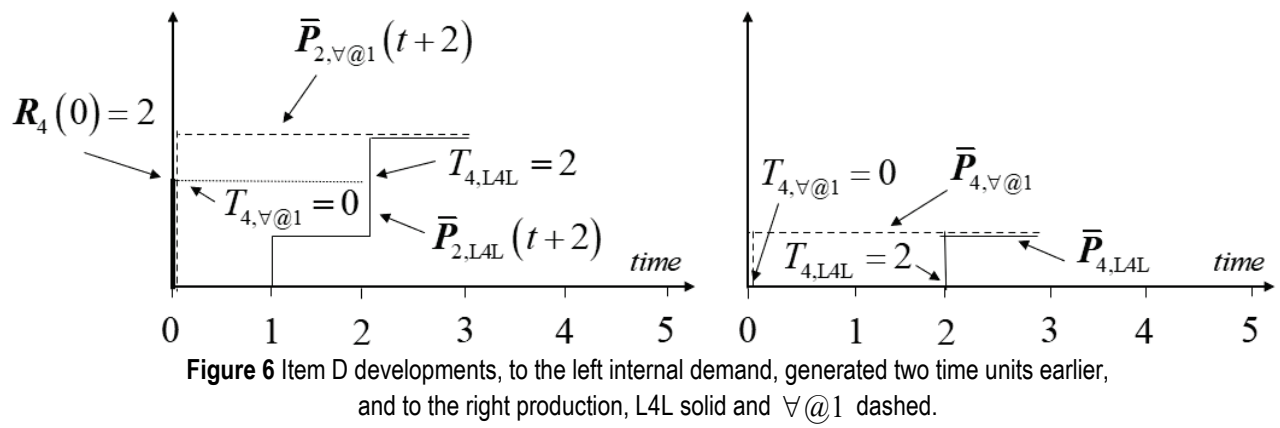


We now turn to economic consequences and assume the following parameter values

Table 1 Assumed economic parameter values

\begin{tabular}{|l|c|l|}
\multicolumn{1}{|c|}{ Tame } & Assumed parameter values & \multicolumn{1}{c|}{ Comments } \\
\hline Sale price vector & $\boldsymbol{p}=[1000,0,0,0]$ & $\begin{array}{l}\text { Only item 1 (A) is } \\
\text { sold externally }\end{array}$ \\
\hline $\begin{array}{l}\text { Variable production } \\
\text { cost vector }\end{array}$ & $\boldsymbol{c}=[200,100,300,200]$ & \\
\cline { 1 - 2 } Setup cost vector & $\boldsymbol{K}=[400,250,300,250]$ & \\
\hline $\begin{array}{l}\text { Continuous discount } \\
\text { rate }\end{array}$ & $\rho=10 \%-30 \%$ & $\begin{array}{l}\text { Consequences are } \\
\text { computed for } \\
\text { different rates }\end{array}$ \\
\hline
\end{tabular}

With the parameter values given in Tab. 1, the following $N P V$ is calculated for our two policies (both for $\rho=0.1$ ):

$\left\{\begin{array}{l}N P V_{\mathrm{L} 4 \mathrm{~L}}=\boldsymbol{p} \tilde{\boldsymbol{D}}(\rho)-\boldsymbol{c} \tilde{\boldsymbol{P}}_{\mathrm{L} 4 \mathrm{~L}}(\rho)-\boldsymbol{K} \tilde{\boldsymbol{P}}_{\mathrm{L} 4 \mathrm{~L}}^{\prime}(\rho)=2425.34 \\ N P V_{\forall @ 1}=\boldsymbol{p} \tilde{\boldsymbol{D}}(\rho)-\boldsymbol{c} \tilde{\boldsymbol{P}}_{\forall @ 1}(\rho)-\boldsymbol{K} \tilde{\boldsymbol{P}}_{\forall @ 1}^{\prime}(\rho)=2902.81\end{array}\right.$

So for $\rho=0.1$, the $\forall @ 1$ policy gives a higher $N P V$ than $\mathrm{L} 4 \mathrm{~L}$ and is to be preferred. However, for higher holding costs, represented by a higher value of $\rho$, one would expect the L4L policy to dominate, since L4L, among all possible policies, keeps available inventory to a very minimum. Varying $\rho$ between 0.1 and 0.3 produces the $N P V$ consequences displayed in Fig. 7.

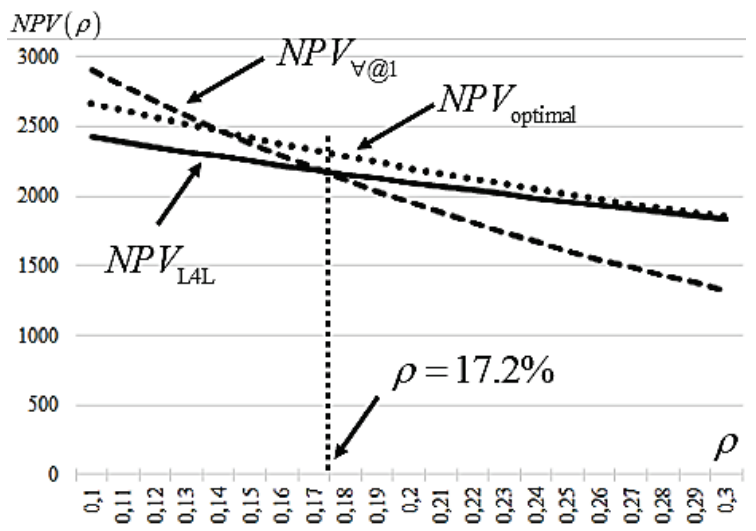

Figure 7 NPV as function of discount rate for policies L4L (solid), $\forall @ 1$ (dashed) and the optimal in-between policy (dotted)

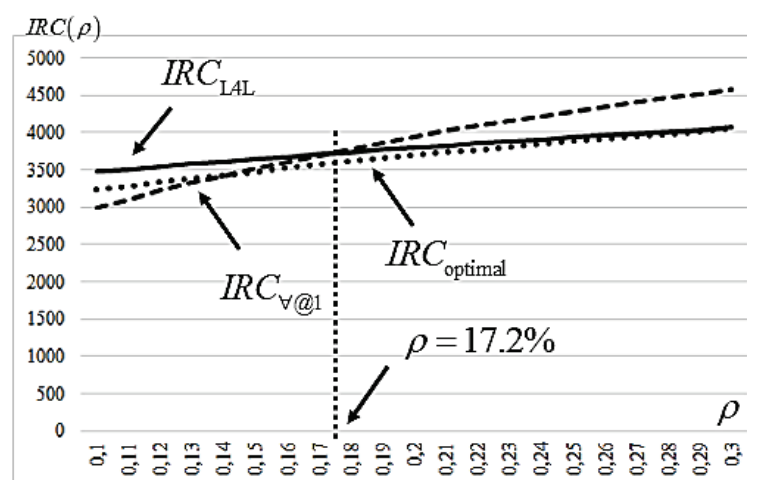

Figure 8 Inventory related costs (IRC) as function of discount rate for policies L4L (solid), $\forall @ 1$ (dashed) and the optimal in-between policy (dotted).
From Figs. 7-8, presenting the same conclusion, we see that for low values of $\rho, \forall @ 1$ (dashed curve) is to be preferred to $\mathrm{L} 4 \mathrm{~L}$ (solid curve) giving a higher $N P V$, and vice versa for values of $\rho$ above $17.2 \%$. At the intersection between the two curves, both policies are equally preferred, but the NPV can be improved further (inventory related costs lowered, see (21)) up (down) to the dotted curve.

There are only 128 different available production plans $\tilde{\boldsymbol{P}}(s)$ available in this example that meet the inner corner requirement for optimality (see e.g. [13] or [31]). For discount rates below $16 \%$, the $\forall @ 1$ policy is optimal, for $\rho$ between $16 \%$ and $26 \%$, the plan

$\tilde{\boldsymbol{P}}(s)=\left[\begin{array}{l}\mathrm{e}^{-3 s}+\mathrm{e}^{-4 s}+2 \mathrm{e}^{-5 s} \\ 3 \mathrm{e}^{-3 s} \\ 2 \mathrm{e}^{-3 s}+4 \mathrm{e}^{-4 s} \\ \mathrm{e}^{-s}\end{array}\right]$ is optimal, and above $26 \%$,

L4L is optimal, but the improvement here is so minute that it does not show in the diagrams.

To determine the value of having an initial inventory, we examine possible plans when $\boldsymbol{R}(0)=\boldsymbol{0}$. Starting with L4L using the Leontief inverse in (34), we find $\tilde{\boldsymbol{P}}_{\mathrm{L} 4 \mathrm{~L}}(s)$ to be

$$
\begin{aligned}
& \tilde{\boldsymbol{P}}_{\mathrm{L} 4 \mathrm{~L}}(s)=(\boldsymbol{I}-\boldsymbol{H} \tilde{\boldsymbol{\tau}}(s))^{-1} \tilde{\boldsymbol{D}}(s)=\left[\begin{array}{cccc}
1 & 0 & 0 & 0 \\
\mathrm{e}^{s} & 1 & 0 & 0 \\
2 \mathrm{e}^{s} & 0 & 1 & 0 \\
\mathrm{e}^{3 s} & \mathrm{e}^{2 s} & 0 & 1
\end{array}\right] \tilde{\boldsymbol{D}}(s)= \\
& =\left[\begin{array}{l}
2 \mathrm{e}^{-s}+\mathrm{e}^{-2 s}+3 \mathrm{e}^{-3 s}+\mathrm{e}^{-4 s}+2 \mathrm{e}^{-5 s} \\
2+\mathrm{e}^{-s}+3 \mathrm{e}^{-2 s}+\mathrm{e}^{-3 s}+2 \mathrm{e}^{-4 s} \\
4+2 \mathrm{e}^{-s}+6 \mathrm{e}^{-2 s}+2 \mathrm{e}^{-3 s}+4 \mathrm{e}^{-4 s} \\
{\left[2 \mathrm{e}^{2 s}+\mathrm{e}^{s}\right]+3+\mathrm{e}^{-s}+2 \mathrm{e}^{-2 s}}
\end{array}\right]
\end{aligned}
$$

We see from the bottom left corner in the right-hand member that production must then take place in past time (market with brackets), so meeting this demand is impossible. Some external demand must be backlogged. The least possible change to remove this problem, is to backlog the first occurring three units of demand by changing external demand from $\left(2 \mathrm{e}^{-s}+\mathrm{e}^{-2 s}+3 \mathrm{e}^{-3 s}+\mathrm{e}^{-4 s}+2 \mathrm{e}^{-5 s}\right)$ to $\left(6 \mathrm{e}^{-3 s}+\mathrm{e}^{-4 s}+2 \mathrm{e}^{-5 s}\right)$. Therefore, the customers will have to wait two periods for two units and one period for one unit, which also postpones revenues. This gives the feasible production plan

$\tilde{\boldsymbol{P}}_{\mathrm{L} 4 \mathrm{~L}}(s)=\left[\begin{array}{l}6 \mathrm{e}^{-3 s}+\mathrm{e}^{-4 s}+2 \mathrm{e}^{-5 s} \\ 6 \mathrm{e}^{-2 s}+\mathrm{e}^{-3 s}+2 \mathrm{e}^{-4 s} \\ 12 \mathrm{e}^{-2 s}+2 \mathrm{e}^{-3 s}+4 \mathrm{e}^{-4 s} \\ 6+\mathrm{e}^{-s}+2 \mathrm{e}^{-2 s}\end{array}\right]$. 
The economic consequences from not having an initial inventory are obtained from computing the NPV of revenues, production and setups. Choosing $\rho=20 \%$, these amount to $N P V_{\text {revenues }}=4477.96, N P V_{\text {production }}=6356.04$ and $N P V_{\text {setups }}=$ 2834.40 , i.e. totalling the negative value of $N P V=-4712.48$. This may be compared to the original $\mathrm{L} 4 \mathrm{~L}$ values (with an initial inventory), which are $N P V_{\text {revenues }}=5139.30$, $N P V_{\text {production }}=1494.07$ and $N P V_{\text {setups }}=1546.75$, totalling $N P V=+2098.48$. The number of setups has increased from eight to twelve. The postponement of revenues causes a loss of 661.35. The difference in $N P V$ with and without the initial inventory amounts to $\triangle N P V=6810.97$, which we can compare with the sales value of the five units of the end item and nominal production cost (disregarding setup costs) for the other items in initial inventory, which amount to 6400.00 .

\section{CONCLUSIONS}

In the foregoing, we have attempted to present consequences from having an initial available stock of items, compared with initially having an empty system. This presentation has been brief due to space requirements. Therefore, particularly aspects on consequences of having initial backlogs (negative inventories) have not been included in this report. Both initial inventories and initial backlogs may be distributed in time. There might be outstanding orders not yet haven been delivered into inventory.

Our main conclusion is that this type of theoretical extension to MRP Theory is achievable, but with the effect of added complications by needing to introduce new concepts, above all the new timing matrix $\tilde{\boldsymbol{T}}(s)$ and the concept of remaining demand $\tilde{\boldsymbol{d}}(s)$.

In future related work, emphasis will be on encompassing initial backlogs and outstanding orders.

\section{Notice}

The paper will be presented at MOTSP $2021-12^{\text {th }}$ International Conference Management of Technology - Step to Sustainable Production, which will take place in Poreč/Porenzo, Istria (Croatia), on September 8-10, 2021. The paper will not be published anywhere else.

\section{REFERENCES}

[1] Grubbström, R. W. \& Tang, O. (2000). An Overview of InputOutput Analysis Applied to Production-Inventory Systems. Economic Systems Research, 12(1), 3-25. https://doi.org/10.1080/095353100111254

[2] Bogataj, D. \& Bogataj, M. (2019). NPV approach to material requirements planning theory - a 50-year review of these research achievements. International Journal of Production Research, 57(15/16), 5137-5153. https://doi.org/10.1080/00207543.2018.1524167

[3] Battini, D., Calzavara, M., Sgarbossa, F., Persona, A. 2017. MRP Theory Supporting Trade-Off Between Investments in Collaborative Robots and Production in Foreign Countries for a Water Pumps Supply Chain. In Zadnik Stirn, L., et al., (Eds), SOR '17 Proceedings, Ljubljana: SDI-SOR, 229-234.
[4] Kovačić, D., Hontoria, E., Ros-McDonnell, L., Bogataj, M. 2015. Location and lead-time perturbations in multi-level assembly systems of perishable goods in Spanish baby food logistics. Central European Journal of Operations Research, 23, 607-623. https://doi.org/10.1007/s10100-014-0372-5

[5] Orlicky, J. A. (1975). Material Requirements Planning, McGraw-Hill, New York, N. Y.

[6] Vazsonyi, A. (1955). The Use of Mathematics in Production and Inventory Control. Management Science, 1(1), 70-85. https://doi.org/10.1287/mnsc.1.1.70

[7] Vazsonyi, A. (1958). Scientific Programming in Business and Industry, Wiley, New York, N.Y.

[8] Koopmans, T. C., (Ed.) (1951). Activity Analysis of Production and Allocation, Wiley, New York, N.Y.

[9] Leontief, W. W. (1928). Die Wirtschaft als Kreislauf. Archiv für Sozialwissenschaft und Sozialpolitik, 60, 577-623.

[10] Leontief, W. W. (1936). Quantitative Input and Output Relations in the Economic Systems of the United States. The Review of Economics and Statistics, 18(3), 105-125. https://doi.org/10.2307/1927837

[11] Grubbström, R. W. \& Ovrin, P. (1992). Intertemporal Generalization of the Relationship between Material Requirements Planning and Input-Output Analysis. International Journal of Production Economics, 26, 311-318. https://doi.org/10.1016/0925-5273(92)90081-H

[12] Bogataj, M. (1999). Inventory allocation and customer travelling problem in spatial duopoly. International Journal of Production Economics, 59, 271-279. https://doi.org/10.1016/S0925-5273(98)00103-0

[13] Bogataj, M. \& Grubbström, R. W. (2012). On the representation of timing for different structures within MRP theory. International Journal of Production Economics, 140, 749-755. https://doi.org/10.1016/j.ijpe.2011.04.016

[14] Bogataj, M. \& Grubbström, R. W. (2013). Transportation delays in reverse logistics. International Journal of Production Economics, 143, 395-402. https://doi.org/10.1016/j.ijpe.2011.12.007

[15] Grubbström, R. W., Bogataj, L., \& Bogataj, M. (2010). Optimal Lotsizing within MRP Theory. Annual Reviews in Control, 34(1), 89-100. https://doi.org/10.1016/j.arcontrol.2010.02.004

[16] Laplace, P.-S. (1799). Traité de Mécanique Céleste, J. B. M. Duprat, Libraire pour les Mathématiques, Paris.

[17] Laplace, P.-S. (1812). Théorie analytique des probabilitiés, $\mathrm{M}^{\mathrm{me}} \mathrm{V}^{\mathrm{e}}$ Courcier, Imprimeur-libraire pour les Mathématiques, Paris.

[18] Grubbström, R. W. (1967). On the application of the Laplace transform to certain economic problems. Management Science, 13, 558-567. https://doi.org/10.1287/mnsc.13.7.558

[19] Grubbström, R. W. (2007). Transform Methodology Applied to Some Inventory Problems. Zeitschrift für Betriebswirtschaft, 77(3), 297-324. https://doi.org/10.1007/s11573-007-0022-7

[20] Grubbström, R. W. (2018). Risk preference evaluation - a fourth dimension of the application of the Laplace transform. International Journal of Production Research, 56(1-2), 344373. https://doi.org/10.1080/00207543.2017.1401248

[21] Grubbström, R. W. (2021). Production decisions based on absolute vs. relative risk aversion and their extensions. International Journal of Production Economics, 234, 108036. https://doi.org/10.1016/j.ijpe.2021.108036

[22] Buser, S. A. (1986). LaPlace Transforms as Present Value Rules: A Note. Journal of Finance, 41, 243-247. https://doi.org/10.1111/j.1540-6261.1986.tb04502.x 
[23] Deakin, M. A. B. (1981). The development of the Laplace transform, 1737-1937: I. Euler to Spitzer, 1737-1880. Archive for History of Exact Sciences, 25, 343-390. https://doi.org/10.1007/BF01395660

[24] Deakin, M. A. B. (1982). The development of the Laplace transform, 1737-1937: II. Poincaré to Doetsch, 1880-1937. Archive for History of Exact Sciences, 25, 351-381. https://doi.org/10.1007/BF00418754

[25] Deakin, M. A. B. (1992). The ascendancy of the Laplace transform and how it came about. Archive for History of Exact Sciences, 44, 265-286. https://doi.org/10.1007/BF00377050

[26] Aseltine, J. A. (1958). Transform Method in Linear System Analysis, McGraw-Hill, New York, N.Y.

[27] Churchill, R. V. (1958). Operational Mathematics, McGrawHill, New York, N.Y.

[28] Churchill, R. V. (1960). Complex Variables and Applications, McGraw-Hill, New York, N.Y.

[29] Spiegel, M. R. (1965). Laplace transforms, McGraw-Hill, New York, N.Y.

[30] Simon, H. A. (1952). On the application of servomechanism theory in the study of production control. Econometrica, 20, 247-268. https://doi.org/10.2307/1907849

[31] Grubbström, R. W. \& Kingsman, B. G. (2004). Ordering and inventory policies for step changes in the unit item cost: A discounted cash flow approach. Management Science, 50, 253267. https://doi.org/10.1287/mnsc. 1030.0150

[32] Grubbström, R. W. \& Huynh, T. T. T. (2006). Analysis of standard ordering policies within the framework of MRP theory. International Journal of Production Research, 44, 3759-3773. https://doi.org/10.1080/00207540600584839

[33] Grubbström, R. W. \& Tang, O. (2012). The space of solution alternatives in the optimal lot-sizing problem for general assembly systems applying MRP theory. International Journal of Production Economics, 140, 765-777. https://doi.org/10.1016/j.ijpe.2011.01.012

\section{Author's contacts:}

Robert W. Grubbström, Emeritus Professor

Linköping Institute of Technology,

Olaus Magnus Väg,

SE-58183 Linköping, Sweden

robert@grubbstrom.com 